\title{
Value at Risk Models in Indian Markets: A Predictive Ability Evaluation Study
}

\author{
Kushagra Goel1, Sunny Oswal2* \\ ${ }^{1}$ Faculty of Management, NMIMS University, Mumbai, India \\ ${ }^{2}$ NMIMS University, Mumbai, India \\ Email: kushagra.goel@nmims.edu, ^sunny.oswal@nmims.edu
}

How to cite this paper: Goel, K. and Oswal, S. (2019) Value at Risk Models in Indian Markets: A Predictive Ability Evaluation Study. Theoretical Economics Letters, 9, 2824-2838.

https://doi.org/10.4236/tel.2019.98177

Received: October 18, 2019

Accepted: December 6, 2019

Published: December 9, 2019

Copyright $\odot 2019$ by author(s) and Scientific Research Publishing Inc. This work is licensed under the Creative Commons Attribution International License (CC BY 4.0).

http://creativecommons.org/licenses/by/4.0/

\begin{abstract}
Value at risk (VaR) is a method of measuring the potential loss in portfolio value for a given distribution of historical returns over a given time period. Measurement of risk therefore becomes essential for a corporate decision. This study attempts to rank the overall predictive ability of select value at risk models in estimating market risks of Indian financial markets. This study estimates the respective predictive ability by employing numerical and graphical measures. The findings plug the gaps in the literature and estimate the best method to be used in the industry. The results evidentially prove that parametric model using normal distribution with GARCH $(1,1)$ fits best for estimating value at risk.
\end{abstract}

\section{Keywords}

Value-at-Risk, Monte Carlo Simulation, Implied Volatility, Historical Based Approach

\section{Introduction}

Risk is an important element when evaluating the effectiveness of business operations. A Risk management plan can help a firm identify future losses, operational inefficiencies, reduce uncertainty and ultimately provide a healthier bottom line. Due to increased global competition, increasing regulations, financial engineering leading to development of complicated securitisation and derivative product, risk management is gaining huge importance. One of the most important steps in risk management is risk measurement. Risk is measured using some common tools such as standard deviation, beta, value at risk and conditional value at risk or sophisticated risk models can also be developed for better results. 


\subsection{Value at Risk (VaR)}

Value at risk $(\mathrm{VaR})$ is a method of measuring the potential loss in portfolio value for a given distribution of historical returns over a given time period.

The maximum possible periodic loss under normal circumstances at a certain confidence level is represented by VaR i.e. Value at Risk. If the loss exceeds VaR, the expected loss is known as Expected Shortfall (ES) or Conditional Value at Risk (CVaR).

Like volatility, with respect to a long term basis, VaR may be extrapolated by multiplying it by the square root of the number of days (i.e. the square root rule). Illustratively, if the daily $\mathrm{VaR}$ is to be converted to annual $\mathrm{VaR}$, multiply the daily VaR by the square root of 252 (considering 252 trading days in a year).

\subsection{Scope}

The study aims to identify, measure and predict market risk for positions in three types of markets viz, Stock Indices, Commodities and Exchange rates. The research intends to use various parametric and nonparametric models to forecast the Value at Risk and also answers why market risk assessment and management are essential for financial institutions.

\subsection{Research Tasks}

1) Analysis and forecasting of one-day value at risk for positions in Nifty 50 Index, INR/USD and gold bullion.

2) Determining the accuracy of four VaR models viz. SMA, EWMA, GARCH and Historical Simulation, in predicting one-day Value at Risk.

3) To rank the overall predictive ability of select value at risk models in estimating Market Risks of Indian Financial Markets.

The above objectives were researched by extensive study performed at NMIMS University, Mumbai.

\section{Review of Literature}

Economic and financial activities globally have been impacting the NASDAQ composite since the last one decade. The paper by [1] KeithKuester, (2006) compared the performance of various existing approaches and some new models for predicting value-at-risk (VaR) in a univariate context. A hybrid method, combining a heavy-tailed generalized autoregressive conditionally heteroskedastic (GARCH) filter with an extreme value theory-based approach exhibited the best results when applied on 30 years of the daily return data on the NASDAQ Composite Index. Also, a new model based on heteroskedastic mixture distributions showed suitable performance. An extension to a particular Conditional autoregressive VaR (CAVaR) model was provided as most CAVaR models perform inadequately.

The paper by [2] Jimmy Skoglund, Donald Erdman, Wei Chen, (2010) evaluates risk models by backtesting the performance of VaR models in predicting 
future losses for a portfolio of seven stocks, futures and options form April 6, 2001 to June 17, 2009. The models underestimate the risk observed during 2008 crisis period quite severely. The assumption on the distribution of univariate GARCH model residual and the choice of copula affect the VaR model performance.

The paper by [3] Chen et al., (2017) proposes a semivariance method for diversified portfolio selection, in which the security returns are given subjective to experts' estimations and depicted as uncertain variables. In the paper, three properties of the semivariance of uncertain variables are verified. Another paper by [4] Zhang et al., (2015) discusses portfolio selection problem in uncertain environment in which security returns cannot be well reflected by historical data, but can be evaluated by the experts.

[5] Koutsoyiannis, A. (2006) in his paper estimated volatility of gold bullion return series. To gauge its predictive ability, twelve different specifications of GARCH models, four different specifications of EGARCH models and four specifications of GJR models were assessed. It was found that both EGARCH specifications and GJR specifications of models are not appropriate for measuring volatility in gold return series. However, the forecasting ability of $\operatorname{GARCH}(3,3)$ is concluded to be more suitable when compared with other GARCH models and with Artificial Neural Networks (ANN) and regression models.

The work done by [6] Shashi Gupta, (2018) examines that the Indian commodity derivative market shows a presence of persistence, mean reversion and leverage effect in its volatility. Augmented EGARCH models were used to measure this volatility with volume and open interest as explanatory variable. Volume showed significant coefficient values whereas open interest failed to show any significant information about the market. Also, time to maturity did not have a significant impact. The statistical tests performed support both, the commodity indices as well as the individual commodities.

[7] Ramazan Gencay, Faruk Selcuk, (2004) investigates the performance of Value at Risk (VaR) approaches applied to the daily index returns of nine emerging markets. The study uses variance-covariance method and historical simulation to get the VaR limits at $99 \%$ and $95 \%$ confidence intervals. In addition, to estimate the tail risk the extreme value theory (EVT) approach is used for stress testing purposes. Since the emerging markets are subject to frequent structural changes, sliding window of Generalized Pareto Distribution (GDP) fits the tails of the return distributions. The EVT results dominate the other $\mathrm{VaR}$ models as it captures the tail risk as well as the dynamic nature of the economy.

In the work done by [8] Zhichao Zhang and Hongyu Pan, (2006), the authors forecast daily volatilities of Shanghai stock exchange composite index (SHZH) and Shenzhen stock exchange composite index (SZZH) using eight different models Random walk, Historical mean, Moving average, Exponential Moving average and four GARCH-type models: GARCH, GJR, EGARCH and APARCH. 
[9] Alper Ozun, Sait Yilmazer, (2010) evaluates eight extreme value theory (EVT) modelling techniques to forecast value at risk (VaR) for the Istanbul Stock Exchange. EVT analyses the extremes in the returns at different quantiles. The lag length for conditional quantile days can be estimated based on the forecasting performance. The models used for back testing are root mean squared error (RMSE), h-step ahead forecasting RMSE, Lopez test, Christoffersen test, Diebold and Mariano test and Kupiec test. The results show that extreme value theory (EVT) performs better than the parametric models as it focuses on the fat tail risk as well.

[10] Timotheos. A, (2010), in the research, analyze the behaviour of the risk management techniques and models for both long and short VaR trading positions. The study investigates three markets form the period of January 3rd 1989 to June 30th 2003. No single model seems to provide statistically acceptable value at risk (VaR) estimate for all securities. However, the forecasting ability of parametric model under GARCH produces comparatively better results.

The seminal work by [11] Alfred Lehar, Christian Schittenkopf and Martin Scheicher, (2002) compares the Black-Scholes framework, namely GARCH and Stochastic Volatility (SV) option pricing model performances in order to eventually estimate the Value at Risk (VaR) for an options portfolio. The models are applied to UK's Financial Times-Stock Exchange 100 Index (FTSE 100) option prices. GARCH clearly dominates stochastic volatility and shows significant overall improvements in pricing performance.

[12] Walsh M. David and Tsou Yu-Gen Glenn, (1998) compare the historical volatility model, an improved extreme-value method (IEV), ARCH/GARCH class of models and an EWMA model of volatility. The data used included the three price indices collected every five minutes from 1 January 1993 to 31 December 1995. The hourly data analysis showed the EWMA and GARCH $(1,1)$ techniques to be the best predictors, depending upon the loss function used, though the difference between them being very slight. The results for the daily data were also same, thereby creating difficulties in identifying the better one between EWMA and GARCH. The weekly data results indicated that EWMA was the best predictor for weekly volatility.

A comparative study by [13] Yu Jun, (2002) evaluates the performance of 9 alternative models for predicting stock price volatility using daily New Zealand data from the period 1980 to 1998. Evaluation method: root mean square error (RMSE), the mean absolute error (MAE), the Theil-U statistics and the LINEX loss function. The RMSE statistics indicated that the SV model provided the most accurate forecast. Despite its simplicity, the random walk model was not found to be a very good forecaster according to RMSE. The MAE statistics favoured the exponential smoothing model, while the SV model came as the second-ranked. Under the Theil-U statistics, SV model again was the best and only one model (i.e. ARCH model) performs worse than the random walk model. When "a" was assigned a value of 10 the LINEX loss function identified SV as the best. With a value of -10 and -20 also LINEX loss function again selected SV 
as the best performer.

[14] Angelidis et al., (2003) explains the applications of GARCH models in VaR estimation. The study estimates Value at Risk (VaR) of perfectly diversified portfolios in five stock indices, using various sample sizes and a number of distributional assumptions.

A computational study by [15] Rockafellar, R., \& Uryasev, S. (2002) attempts to analyse if $\mathrm{CVaR}$ is able to quantify dangers beyond $\mathrm{VaR}$ and moreover if it is coherent. It provides optimization short-cuts which, through linear programming techniques, make practical many large-scale calculations that could otherwise be out of reach. Out of the four models utilized viz. Historical Value at Risk (VaR), Geometric Brownian Motion (GBM), Extreme Value Theory (EVT) and Semi parametric model, EVT depicted best results while Historical Value at Risk $(\mathrm{VaR})$ model was the worst performing one.

Multiple seminal works have contributed to this domain. This study adds to the body of knowledge by plugging the conceptual gap of evaluative the predictive ability of the VaR models by measures that are numerical and graphical in nature.

\section{Approach and Methodology}

\subsection{Data Period}

The study investigates three markets: equity, currency and commodity for a period of 20 years so as to incorporate different economic conditions. There are a total of 4981, 5281 and 5081 observations for Nifty 50 returns, INR/USD returns and gold returns respectively (Table 1 ).

The daily close price for each of the asset is considered as the input to the various models. The close prices are then converted into lognormal returns using the following equation:

$$
R=l(R t / R t-1) \times 100
$$

where,

$$
\begin{aligned}
& R=\text { Return of the closing prices. } \\
& R t=\text { Today's closing prices. } \\
& R t-1=\text { Previous day's closing prices. } \\
& \mathrm{Ln}=\log \text { normal. }
\end{aligned}
$$

\subsection{Sources of Data}

The secondary sources of data used in this study are obtained from NSE, BSE, RBI and Bloomberg database amongst others.

\subsection{Various Approaches for Estimating VaR}

The VaR Estimation Methodologies have two types of classification (Figure 1).

The first classification is based on the type of portfolio position or exposure. A portfolio may have linear exposures like stocks, forwards and futures or non-linear exposures such as options, embedded options and structured products. 
Table 1. Data period for the study.

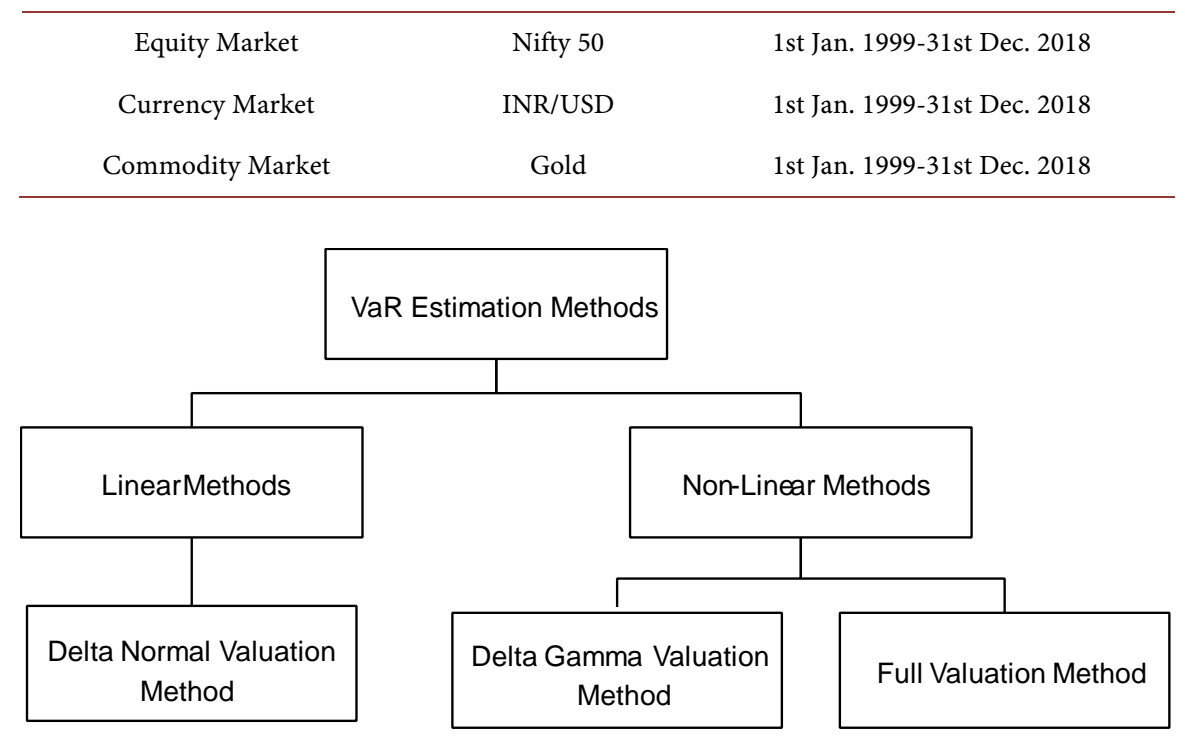

Figure 1. VaR estimation methodologies classification I. (Source: Author Generated).

The Delta Normal Valuation Method is to be used in case of linear derivative such as forwards, Futures and swaps.

The Delta Gamma Valuation Method is to be used in case of non-linear derivatives which are well behaved like options and non-option embedded bonds.

The Full Revaluation Method is to be used in case of non-linear misbehaved series like option embedded bonds i.e. callable bonds or puttable bonds and for structured products like Mortgage Backed Securities (MBS). It is also to be used when there exist cross partial effects (Figure 2).

The second type of classification is based on the type of data used for calculating VaR. Historical based approaches use historical data to calculate the volatility for VaR while the implied volatility approach uses option price and Black and Scholes Model (BSM) to back calculate the forward looking implied volatility.

\subsubsection{Parametric Approaches}

Parametric Approaches are based on the assumption that the asset returns follow a certain distribution, say normal distribution. It is also known as Delta Normal Method, Analytical Approach or Variance Co-Variance (VCV) Approach.

1) Why volatility is required for calculating VaR:

Suppose we have a portfolio whose returns are normally distributed with a daily mean $=0$ and daily volatility $=1.5 \%$. The portfolio is currently worth $\$ 1000$ million. The 1-day VaR at $99 \%$ confidence interval is as follows:

For an area of $1 \%$ in a tail $\mathrm{z}=2.33$.

$\operatorname{VaR}(\%)=z^{\star} \sigma=2.33 * 1.5 \%=3.5 \%$.

$\mathrm{VaR}$ (in $\$$ terms) $=3.5 \%$ of $\$ 1000$ million $=\$ 35$ million.

Thus, maximum possible loss that can take place in a day is $\$ 35$ million with $99 \%$ confidence i.e. with just $1 \%$ chance of being exceeded. In $1 \%$ of the worst cases, loss would be at least $\$ 35$ million. 


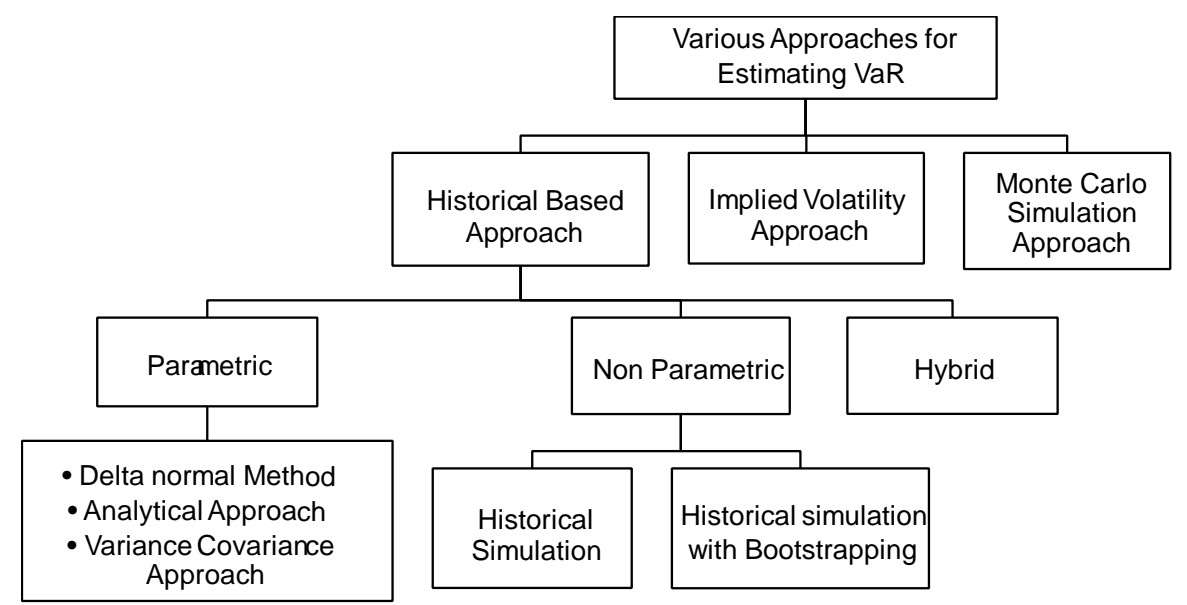

Figure 2. VaR estimation methodologies classification II. (Source: Author Generated.)

The above example goes to show that the standard deviation is a necessary input for estimating the Value at Risk.

2) Observed properties of volatility

A volatility estimation engine must capture the following three properties of volatility:

a) Adaptability: Volatility is dynamic or regime switching so the engine should keep on forecasting new volatility based on innovation.

b) Persistence: Volatility is persistent or sticky, i.e. it tends to cluster around the current value.

c) Mean Reverting: Volatility tends to revert back to a particular mean reversion level.

3) Parameter Estimation (MLE) and choice of window size

Parameter Estimation Techniques

The technique is designed in such a manner that we try to maximize the probability of the observations occurring. The Maximum Likelihood Estimation technique is used in the study for parameter calibration of the various probability distributions considered in the VaR models.

Choice of window size

There is a trade off between statistical accuracy i.e. precision and adaptability when selecting a window size for a volatility estimation engine.

The study uses 1000 days window size to incorporate persistency. The VaR is estimated using approximately past 4 years data.

4) Method 1: VaR using SMA

The simple moving average is also known as the historical standard deviation approach. The unbiased formula for calculation variance under the SMA approach is as follows:

$$
\sigma^{2}=\sum \mu^{2} / k
$$

where, $\mu=$ Past Returns $k$-window size.

5) Method 2: VaR using EWMA 
Variance under EWMA (Exponential weighted moving average) is given by the following equation:

$$
\sigma n 2=\lambda * \sigma 2 n-1+(1-\lambda) * \mu 2 n-1
$$

where, $\mu=$ Past Returns.

$\lambda=$ Decay Factor.

The beauty of EWMA is the requirement of less storage. We just need to store the previous day's estimate of volatility i.e. $\sigma n-1$ and the recent innovation on-1.

The RiskMetrics approach is just an EWMA model that uses a pre-specified decay factor for daily data, which is equal to 0.94 and a factor equal to 0.97 for monthly data.

6) Method 3: VaR using GARCH

One of the most popular methods of estimating volatility is the generalized autoregressive conditional heteroskedastic (GARCH) $(1,1)$ model. The best way to describe GARCH $(1,1)$ model is to take a look at the formula:

$$
\sigma n^{2}=\omega+\alpha \mu^{2} n-1+\beta \sigma^{2} n-1
$$

where:

$\alpha=$ weight on the previous period's return $\beta=$ weight on the previous volatility estimate $\omega=$ weighted long-run variance $=$ VVL.

$V L=$ long-run average variance $=\omega /(1-\alpha-\beta) \alpha+\beta+\mathrm{V}=1 \alpha+\beta<1$ for stability so that $\mathrm{V}$ is not negative.

If $\alpha+\beta>1$ then $\gamma<0$, then GARCH model becomes unstable, as the variance becomes mean fleeing then compared to mean reverting.

7) Analogy between EWMA with GARCH

- EWMA (Exponential weighted moving average) is a special case of GARCH when $\alpha+\beta=1$.

- Conceptually GARCH is better than EWMA as it captures mean reverting tendency, which EWMA approach doesn't.

- EWMA is considered better than GARCH, as it is parsimonious. Requires just one parameter estimation i.e. $\lambda$. Whereas GARCH requires three parameters $\alpha, \beta$ and $\omega$.

- However, empirically it was found that GARCH performed better than EWMA for each asset class under consideration (Figure 3 ).

\subsubsection{Non Parametric Approaches}

1) Historical Simulation

Historical Simulation does not assume any particular distribution. It also does not suffer from the problem of fat tails as it considers the extreme values of the returns as well.

The following steps were performed to estimate VaR under HS approach:

Step 1: Collected past returns of each asset class.

Step 2: Arranged them from worst to best.

Step 3: Sliced 1\%, 5\% and 10\% from the worst side. 
Projected Volatility

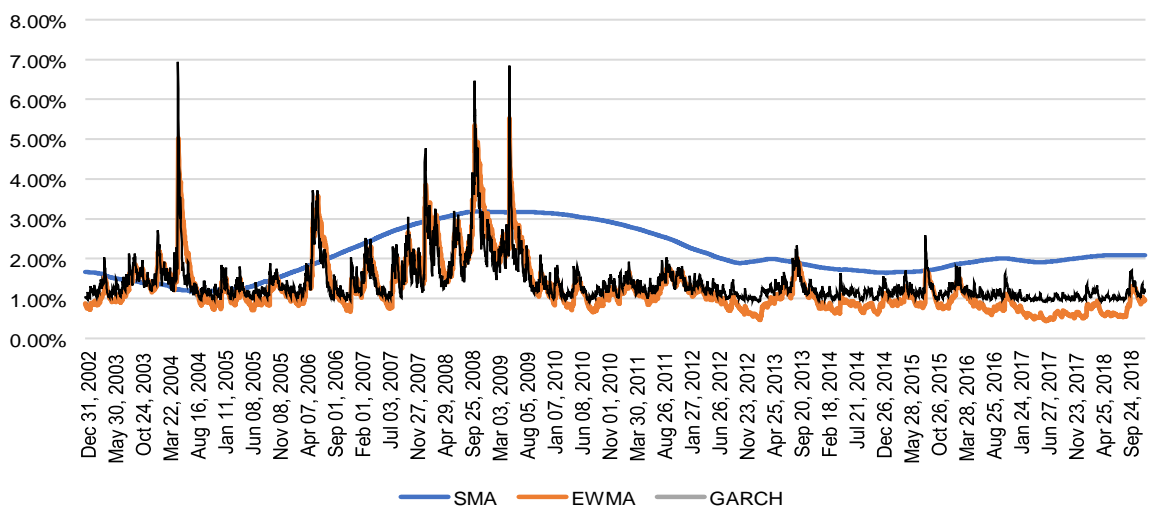

Figure 3. Comparison of the three volatility estimation engines. (Source: Author Generated).

2) Historical Simulation with Bootstrapping

Historical Simulation with Bootstrapping is an improved approach of Historical Simulation approach. It involves random sampling from past observations with replacement. We then arrange the data from worst to best. To get the $\mathrm{VaR}$, we slice the significance level percentage from the worst side.

\section{Analysis and Findings}

This study employs both numerical measures and graphical measures to evaluate the predictive ability of the VaR models as stated in the previous section. Multiple analytical techniques as mentioned in the figure below may be employed (Figure 4).

\subsection{Descriptive Analysis}

Descriptive analysis involves describing the characteristics of data i.e. summarising the data.

a) Numerical Measures (Table 2)

b) Graphical Measures:

The figure (Figure 5) reflects daily Nifty 50 Index from $1^{\text {st }}$ Jan 1999 to $31^{\text {st }}$ Dec 2018. The horizontal axis corresponds to time while the vertical axis displays the value of the index.

Nifty 50 log returns reflect a subdued volatility over 20 years. The stationarity and volatility clustering in the market is evident (Figure 6).

The above figure (Figure 7) portrays the daily INR/USD from $1^{\text {st }}$ Jan 1999 to $31^{\text {st }}$ Dec 2018. The horizontal axis corresponds to time while the vertical axis displays the closing exchange rate.

The above figure reflects the INR/USD log returns. The stationarity and volatility clustering in the market is visible (Figure 8).

The daily gold close price from $1^{\text {st }}$ Jan 1999 to $31^{\text {st }}$ Dec 2018 is chalked in the figure above (Figure 9). The horizontal axis corresponds to time while the vertical axis displays gold close price. 


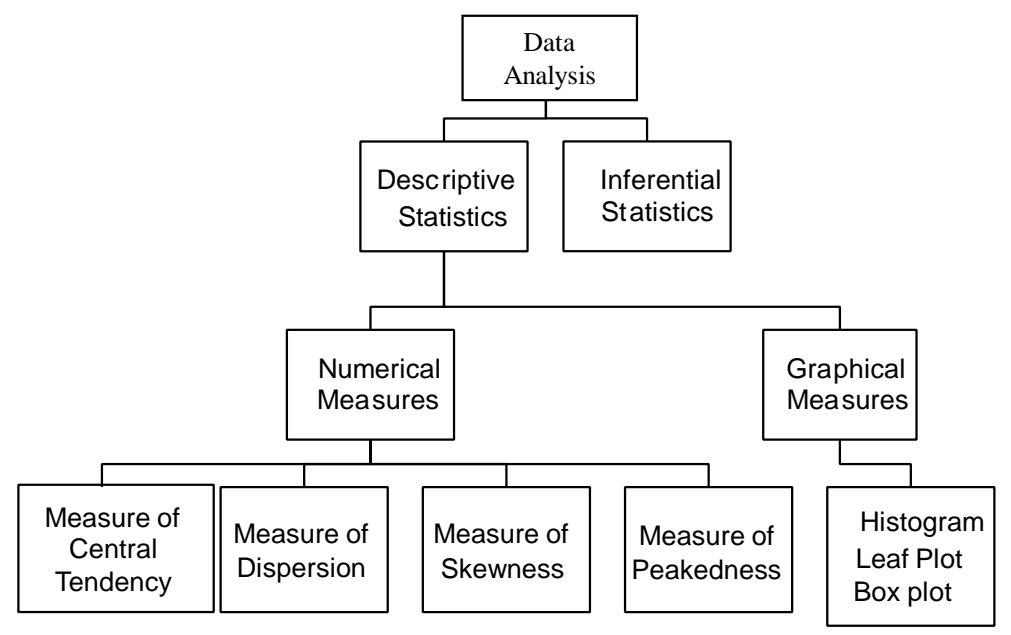

Figure 4. Data Analysis techniques for VaR Model prediction (Source: Author Generated).

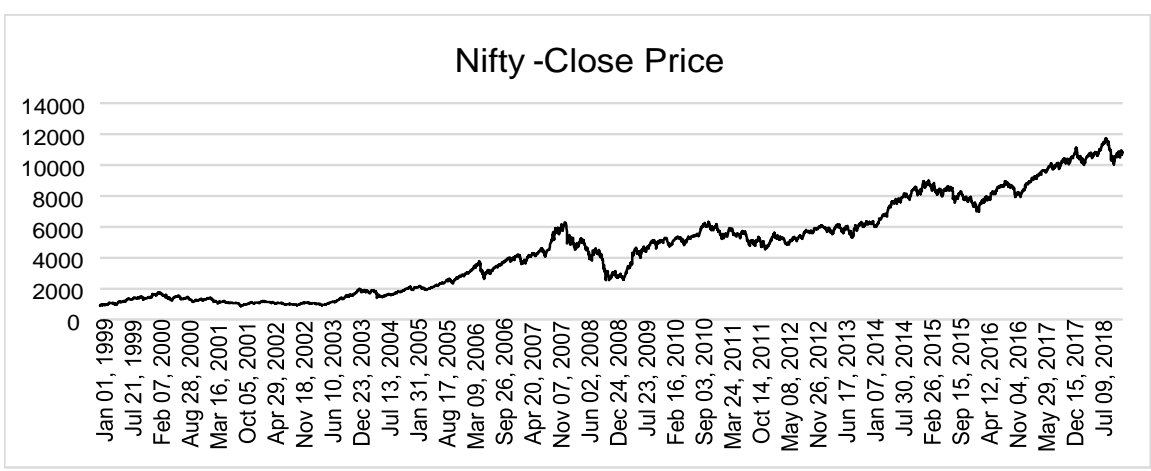

Figure 5. Nifty 50 historical data (Source: Author Generated).



Figure 6. Nifty 50 Log returns (Source: Author Generated).

Even ingold returns, the stationarity and volatility clustering in the market areee visible (Figure 10).

\subsection{Unit Root Analysis}

Unit root analysis is tested using the Augmented Dickey-Fuller (ADF) test. To test for stationary, we conducted the ADF test. It is used for larger and more complicated set of time series models. We can perform GARCH forecasting only on stationary data. Thus, the data needs to be made stationary. 
Table 2. Numerical measures (Consolidated).

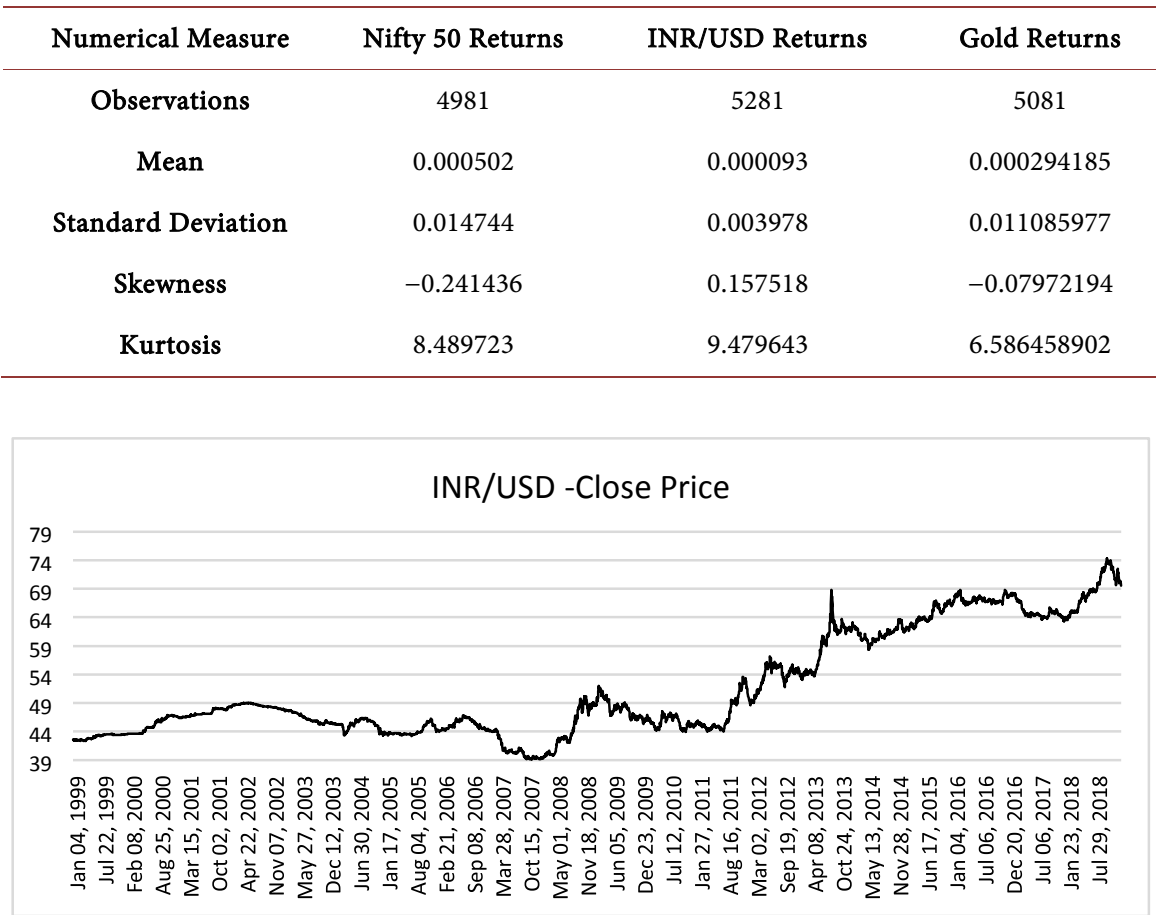

Figure 7. INR/USD historical data (Source: Author Generated).

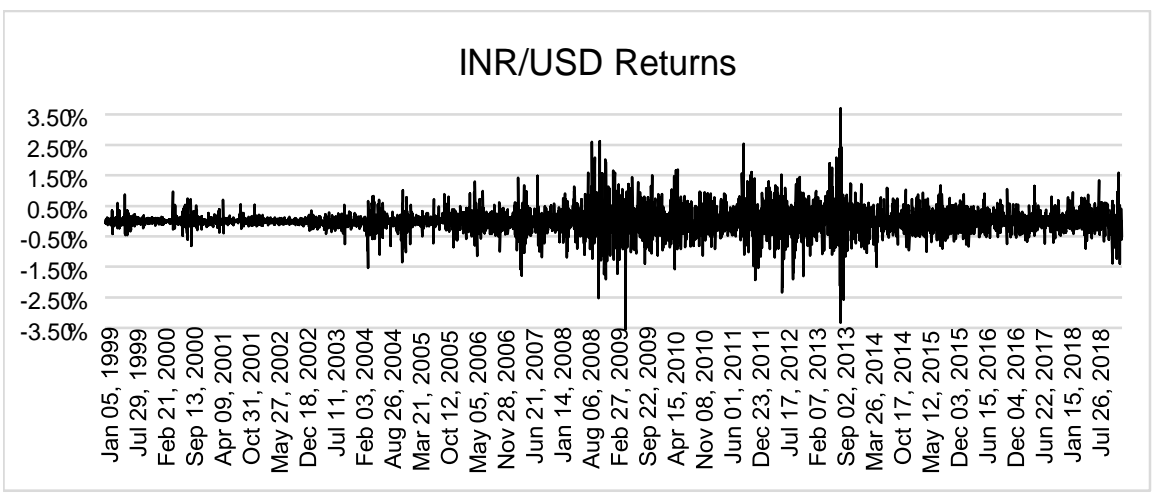

Figure 8. INR/USD Log returns (Source: Author Generated).

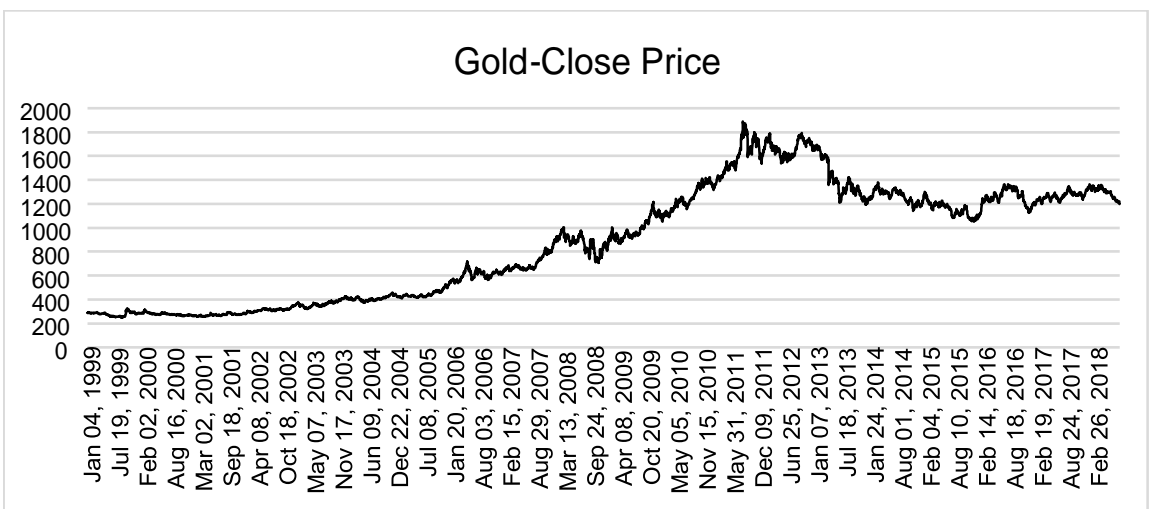

Figure 9. Commoditized Gold historical data (Source: Author Generated). 


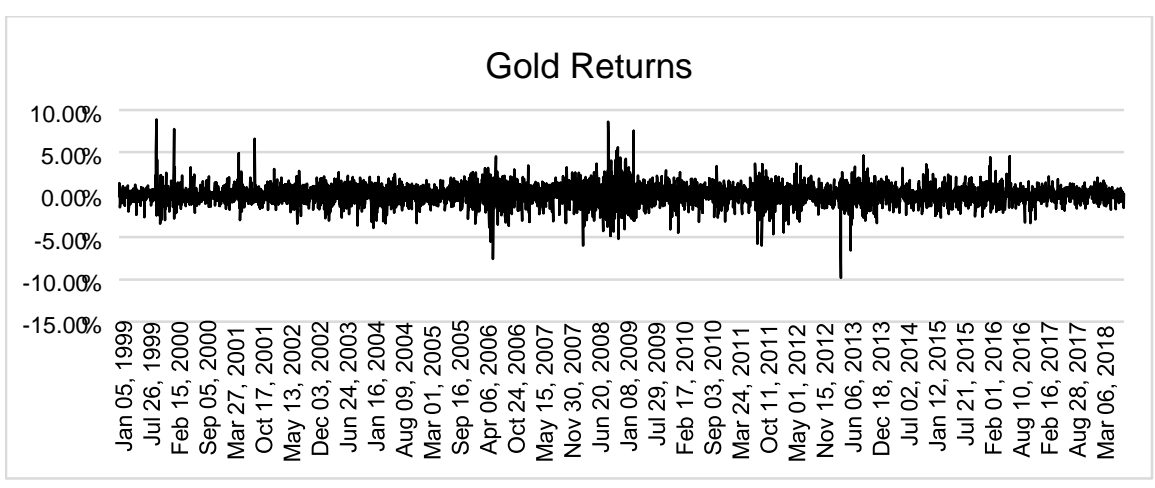

Figure 10. Commoditized Gold log returns (Source: Author Generated).

When having a unit root $\rho 1=1$ in the following equation

$$
Y t=\rho 1 Y t-1+\rho 2 \Delta Y t-1+\rho 3 \Delta Y t-2+\varepsilon t
$$

This is called the augmented Dickey-Fuller (ADF) test and implemented in many statistical and econometric software packages. We have conducted ADF test on Excel through Real Stat software package.

\subsection{Back-Testing Results with Exceedances}

Back testing is the process of comparing losses predicted by the value at risk (VaR) model to those actually experienced over the sample testing period.

If a model were completely accurate, we would expect VaR to be exceeded (also called as an exception) with the same frequency predicted by the confidence level used in the VaR model. In other words, the probability of observing a loss amount greater than $\mathrm{VaR}$ is equal to the significance level.

When using the above back testing approach, the following three desirable attributes of VaR estimates are to be evaluated: VaR estimate should be unbiased, adaptable and robust.

\section{Conclusions}

\subsection{Inferences}

- The results show that parametric model using normal distribution with GARCH $(1,1)$ fits best for estimating value at risk at $99 \%$ confidence interval in all the three markets under consideration.

- The research indicates that the Historical simulation model with $99 \%$ confidence fails the backtesting test for each asset class.

\subsection{Implications}

- The findings will assist the commercial and investment banks in determining the extent and occurrence ratio of potential losses in their institutional portfolios.

- The study will help build additional confidence in measuring the extent of potential forced reductions of the firm's capital over short time periods. 


\subsection{Limitations}

- The statistical probability distributions assumed for testing purposes are limited to normal and student-t distributions only.

- The calculations done are to estimate only 1-day VaR. However, the study can be extended to estimate 30-day VaR, 50-day VaR, 100 day-VaR according to the investors' need and risk exposure.

- The research studies only the market risk in the Indian markets. The VaR concept can be applied to estimate the credit risk, operational risk and liquidity risk.

- The rolling window size considered for backtesting is of 1000 days. Rolling window sizes play a crucial role in determining the $\mathrm{VaR}$ as it leads to a choice between persistence and adaptability.

- The study considers three markets viz. index, exchange rate and commodities. The models can be used for individual stocks as well for derivative instruments like futures and options.

\section{Conflicts of Interest}

The authors declare no conflicts of interest regarding the publication of this paper.

\section{References}

[1] Kuester, K. (2006) Value-at-Risk Prediction: A Comparison of Alternative Strategies. Journal of Financial Econometrics, 4, 53-89. https://doi.org/10.1093/jifinec/nbj002

[2] Skoglund, J., Erdman, D. and Chen, W. (2010) The Performance of Value-at-Risk Models during the Crisis. The Journal of Risk Model Validation, 4, 3-21. https://doi.org/10.21314/JRMV.2010.052

[3] Chen, L., Peng, J., Zhang, B. and Rosyida, I. (2017) Diversified Models for Portfolio Selection Based on Uncertain Semivariance. International Journal of Systems Science, 48, 637-648. https://doi.org/10.1080/00207721.2016.1206985

[4] Zhang, B., Peng, J. and Li, S. (2015) Uncertain Programming Models for Portfolio Selection with Uncertain Returns. International Journal of Systems Science, 46, 2510-2519. https://doi.org/10.1080/00207721.2013.871366

[5] Koutsoyiannis, A. (2006) A Short-Run Pricing Model for a Speculative Asset, Tested with Data from the Gold Bullion Market. Applied Economics, 15, 563-581. https://doi.org/10.1080/00036848300000037

[6] Gupta, S., Choudhary, H. and Agarwal, D.R. (2018) An Empirical Analysis of Market Efficiency and Price Discovery in Indian Commodity Market. Global Business Review, 19, 771-789. https://doi.org/10.1177/0972150917713882

[7] Gencay, R. and Selçuk, F. (2004) Extreme Value Theory and Value-at-Risk: Relative Performance in Emerging Markets. International Journal of Forecasting, 20, 287-303. https://doi.org/10.1016/j.ijforecast.2003.09.005

[8] Zhang, Z. and Pan, H. (2006) Forecasting Financial Volatility: Evidence from Chinese Stock Market. SSRN Electronic Journal. https://doi.org/10.2139/ssrn.903937

[9] Ozun, A. and Yilmazer, S. (2010) Filtered Extreme-Value Theory for Value-at-Risk 
Estimation: Evidence from Turkey. The Journal of Risk Finance, 11, 164-179. https://doi.org/10.1108/15265941011025189

[10] Timotheos, A. (2010) Idiosyncratic Risk in Emerging Markets. Financial Review, 45, 1053-1078. https://doi.org/10.1111/j.1540-6288.2010.00285.x

[11] Lehar, A., Scheicher, M. and Schittenkopf, C. (2002) GARCH vs. Stochastic Volatility: Option Pricing and Risk Management. Journal of Banking \& Finance, 26, 323-345. https://doi.org/10.1016/S0378-4266(01)00225-4

[12] Walsh, D.M. and Tsou, G.Y.-G. (1998) Forecasting Index Volatility: Sampling Interval and Non-Trading Effects. Applied Financial Economics, 8, 477-485. https://doi.org/10.1080/096031098332772

[13] Yu, J. (2002) Forecasting Volatility in the New Zealand Stock Market. Applied Financial Economics, 12, 193-202. https://doi.org/10.1080/09603100110090118

[14] Angelidis, T., Benos, A. and Degiannakis, S. (2003) The Use of GARCH Models in VaR Estimation. Statistical Methodology, 1, 105-128. https://doi.org/10.1016/j.stamet.2004.08.004

[15] Rockafellar, R. and Uryasev, S. (2002) Conditional Value-at-Risk for General Loss Distributions. Journal of Banking \& Finance, 26, 1443-1471.

https://doi.org/10.1016/S0378-4266(02)00271-6 


\section{Appendix}

Rolling EWMA code in R language:

library ("tseries") library ("zoo") library ("forecast") library ("FinTS") library ("rugarch")

Niftydata <- read.csv ("Nifty4981.csv") attach (Niftydata) Nifty.return <- Return result $=$ matrix $(\mathrm{c}(0,0,0,0), \mathrm{ncol}=4) \mathrm{a}=1$ while $(\mathrm{a}<3982)$

$\{b=a+999$

$\mathrm{X}<-$ Nifty.return[a:b] ewma_spec $<-$ ugarchspec (variance.model $=$ list $($ model $=$ "iGARCH", garchOrder $=c(1,1))$, mean $\cdot$ model $=$ list $($ armaOrder $=c$ $(0,0)$, include.mean $=$ TRUE), distribution.model $=$ "norm", fixed.pars $=$ list $($ omega $=0))$ ewma_fit $<-$ ugarchfit $($ spec $=$ ewma_spec, data $=\mathrm{X})$

result = rbind (result,ewma_fit@fit $\$$ coef) $a=a+1\}$ write.csv (result,"result.csv")

Rolling GARCH $(1,1)$ code in R language

library ("tseries") library ("zoo") library ("forecast") library ("FinTS") library ("rugarch") Niftydata<- read.csv ("Nifty4981.csv") attach (Niftydata) Nifty.return $<-$ Return result $=$ matrix $(c(0,0,0,0,0,0)$, ncol $=6) a=1$ while $(\mathrm{a}<3982)$

$\{b=a+999$

$\mathrm{X}<-$ Nifty.return[a:b]

res_garch11_spec $<-$ ugarchspec (variance.model $=$ list $($ garchOrder $=c(1,1))$, mean.model $=$ list $(\operatorname{armaOrder}=\mathrm{c}(1,1)))$ res_garch11_fit $<-$ ugarchfit $(\operatorname{spec}=$ res_garch11_spec, data $=\mathrm{X})$ result = rbind (result, res_garch11_fit@fit $\$$ coef) $\mathrm{a}=$ $\mathrm{a}+1$

\} write.csv (result, "result2.csv") 